\title{
Do Fermi-LAT observations really imply very large Lorentz factors in GRB outflows?
}

\section{Romain Hascoët}

E-mail: hascoeteiap.fr

\section{Vincent Vennin}

\section{Frédéric Daigne*}

\section{Robert Mochkovitch}

UPMC Univ Paris 06, UMR 7095, Institut d'Astrophysique de Paris, F-75014, Paris, France CNRS, UMR 7095, Institut d'Astrophysique de Paris, F-75014, Paris, France

\begin{abstract}
Recent detections of GeV photons in a few GRBs by Fermi-LAT have led to strong constraints on the bulk Lorentz factor in GRB outflows. To avoid a large $\gamma \gamma$ optical depth, minimum values of the Lorentz factor have been estimated to be as high as 800-1200 in some bursts. Here we present a detailed calculation of the $\gamma \gamma$ optical depth taking into account both the geometry and the dynamics of the jet. In the framework of the internal shock model, we compute lightcurves in different energy bands and the corresponding spectrum and we show how the limits on the Lorentz factor can be significantly lowered compared to previous estimates.

Our detailed model of the propagation of high energy photons in GRB outflows is also appropriate to study many other consequences of $\gamma \gamma$ annihilation in GRBs: (i) the $\gamma \gamma$ cutoff transition in a time-integrated spectrum is expected to be closer to a power-law steepening of the spectrum than to a sharp exponential decay; (ii) the temporal evolution of the $\gamma \gamma$ opacity during a burst favors a delay between the $\mathrm{MeV}$ and $\mathrm{GeV}$ light curves; (iii) for complex GRBs, the $\gamma \gamma$ opacity suppress the shortest time-scale features in high energy light curves (above $100 \mathrm{MeV}$ ). Finally we also consider GRB scenarii where $\mathrm{MeV}$ and $\mathrm{GeV}$ photons are not produced at the same location, showing that the $\gamma \gamma$ opacity could be further lowered, reducing even more the constraint on the minimum Lorentz factor.
\end{abstract}

25th Texas Symposium on Relativistic Astrophysics

December 6-10, 2010

Heidelberg, Germany

\footnotetext{
*Institut Universitaire de France
} 


\section{Introduction}

The compactness problem. The short time scales observed in GRBs (down to a few ms) can be used to deduce an upper limit on the size of the emitting region producing $\gamma$-rays. This information combined with the huge isotropic $\gamma$-ray luminosities deduced from the measured redshifts imply huge photon densities. Then the simplest assumption of an emission produced by a plasma radiating isotropically with no macroscopic motion predicts that $\gamma$-ray photons should not escape due to $\gamma \gamma$ annihilation $\gamma \gamma \rightarrow e^{+} e^{-}$. This is in contradiction with the observed GRB spectra which are non-thermal and extend well above the rest-mass electron energy $m_{e} c^{2} \approx 511 \mathrm{keV}$. Observation and theory can be reconciled by assuming that the emitting material is moving at ultra-relativistic velocities [1]. This is mainly due to relativistic beaming. First the relativistic beaming implies that the observer will see only a small fraction of the emitting region: the constraint on the size of the emitting region is now less severe. Second the collimation of photons in the same direction reduce the number of potential interactions. Finally the typical $\gamma \gamma$ interaction angle becoming small the photon energy threshold for pair production becomes higher. This theoretical context combined with the observational data gives the possibility to estimate a minimum Lorentz factor $\Gamma_{\min }$ for the emitting outflow in GRBs [2] (or directly a Lorentz factor estimate if the $\gamma \gamma$ cutoff is clearly identified in the spectrum, see [3]).

Severe constraints on the Lorentz factor from Fermi-LAT observations. Since the launch of Fermi in June 2008, the LAT instrument has detected high energy photons above $10 \mathrm{GeV}$ in a few GRBs. The observed $\gamma$-ray spectrum often remains consistent with a Band function covering the GBM and LAT spectral ranges without any evidence of a high energy cutoff which could be identified as a signature of $\gamma \gamma \rightarrow e^{+} e^{-}$. This extension by Fermi of the observed spectral range upper bound from $10 \mathrm{MeV}$ (e.g. BATSE) to $10 \mathrm{GeV}$ implies constraints on $\Gamma_{\min }$ which are much more severe than the ones obtained previously. In a few cases $\Gamma_{\min }$ has been estimated to be of the order of 1000 (for example: GRB 080916C $-\Gamma_{\min }=887$ [4], GRB $090510-\Gamma_{\min }=1200$ [5]). These extreme values put severe constraints on the physics of the central engine which should be able to strongly limit the baryon load in the outflow.

However these $\Gamma_{\min }$ values were obtained from a simplified "single zone" model where the spatial and temporal dependencies are averaged out. The motivation of this work is to develop a detailed approach taking into account a more realistic treatment of the dynamics.

\section{Computing the $\gamma \gamma$ optical depth}

General $\gamma \gamma$ opacity formula. The $\gamma \gamma$ opacity $\left(\tau_{\gamma \gamma}\right)$ is given by:

$$
\tau_{\gamma \gamma}\left(E_{G e V}\right)=\int_{l_{e}}^{\infty} d l \int d \Omega \int_{E_{c}\left(E_{G e V}, \psi\right)}^{\infty} d E n_{\Omega}(E) \sigma_{\gamma \gamma}(E, \psi)(1-\cos \psi)
$$

All the physical quantities are measured in the laboratory (or source) frame. $E_{G e V}$ is the energy of the photon for which $\tau_{\gamma \gamma}$ is calculated whereas $E$ is the energy of the interacting field photon. $\psi$ represents the interaction angle between the $\mathrm{GeV}$ photon and the interacting photon and $\sigma_{\gamma \gamma}$ is the $\gamma \gamma$ interaction cross-section between these two photons. $E_{c}=2\left(m_{e} c^{2}\right)^{2} /\left[E_{G e V}(1-\cos \psi)\right]$ is 

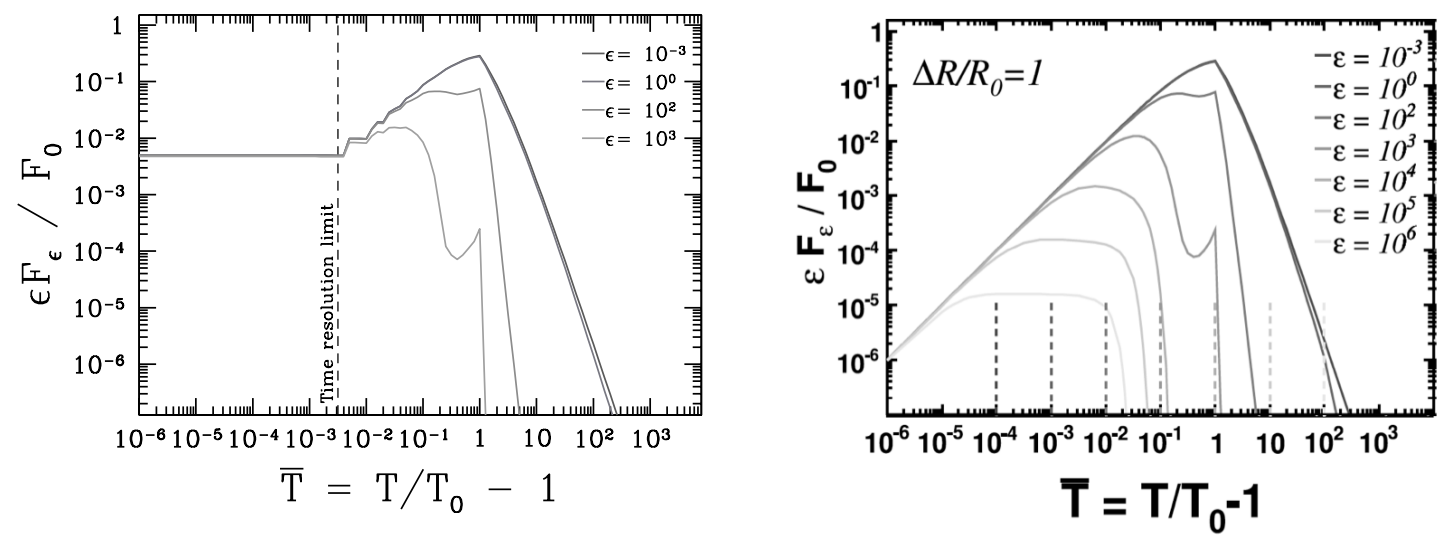

Figure 1: Opacity in a single pulse - comparison with the semi-analytical work by [6]. Left panel: our model. Right panel: model presented in [6]. As illustrated here, our numerical model can reproduce the normalized light-curves shown in [6] (the observer time $\bar{T}$ and observed fluxes $\varepsilon F_{\varepsilon} / F_{0}$ have normalized values). The discrepancy for $\bar{T}<10^{-2}$ is due to numerical resolution limitations (this corresponds to a "true" observer time $t_{o b s}<10^{-4} \mathrm{~s}$ ).

the energy threshold of the field photon above which $\gamma \gamma$ annihilation can happen. Finally $n_{\Omega}$ is the photon field distribution $\left[\mathrm{ph} \cdot \mathrm{cm}^{-3} \cdot \mathrm{erg}^{-1} \cdot \mathrm{sr}^{-1}\right]$ at a given location and time.

The equation (2.1) is made of a triple integral : the $d l$-integration is done over the path of the $\mathrm{GeV}$ photon from its emission location to the observer, the $d \Omega$-integration is done over the solid angle distribution of the interacting photon field surrounding the $\mathrm{GeV}$ photon whereas the $d E$-integration is done over its energy distribution. The equation (2.1) is general and can be applied to any photon emitted at a given location and time with a given propagation direction within the GRB outflow.

Validation of the model. The kernel of our study is the calculation of the $\gamma \gamma$ opacity created by a spherical flash. It is then possible to model the case of a propagating radiating spherical front (representing for example a shock wave) by the succession of many spherical flashes. One of the critical step is the exact calculation of the photon density $n_{\Omega}$ taking into account all the relativistic effects. Before dealing with more complex dynamical configurations within the internal shock framework, the validity of our numerical approach was tested on a simple single-pulse case with a comparison to the previous semi-analytic study of [6] (see fig. 1).

\section{Application to Internal Shocks}

\subsection{Internal shocks within a relativistic outflow}

Now the model is applied to dynamical evolutions expected in the internal shock framework, where the whole prompt $\gamma$-ray emission is produced by electrons accelerated by shock waves propagating within a relativistic variable outflow. We model the dynamics via a multiple shell model 

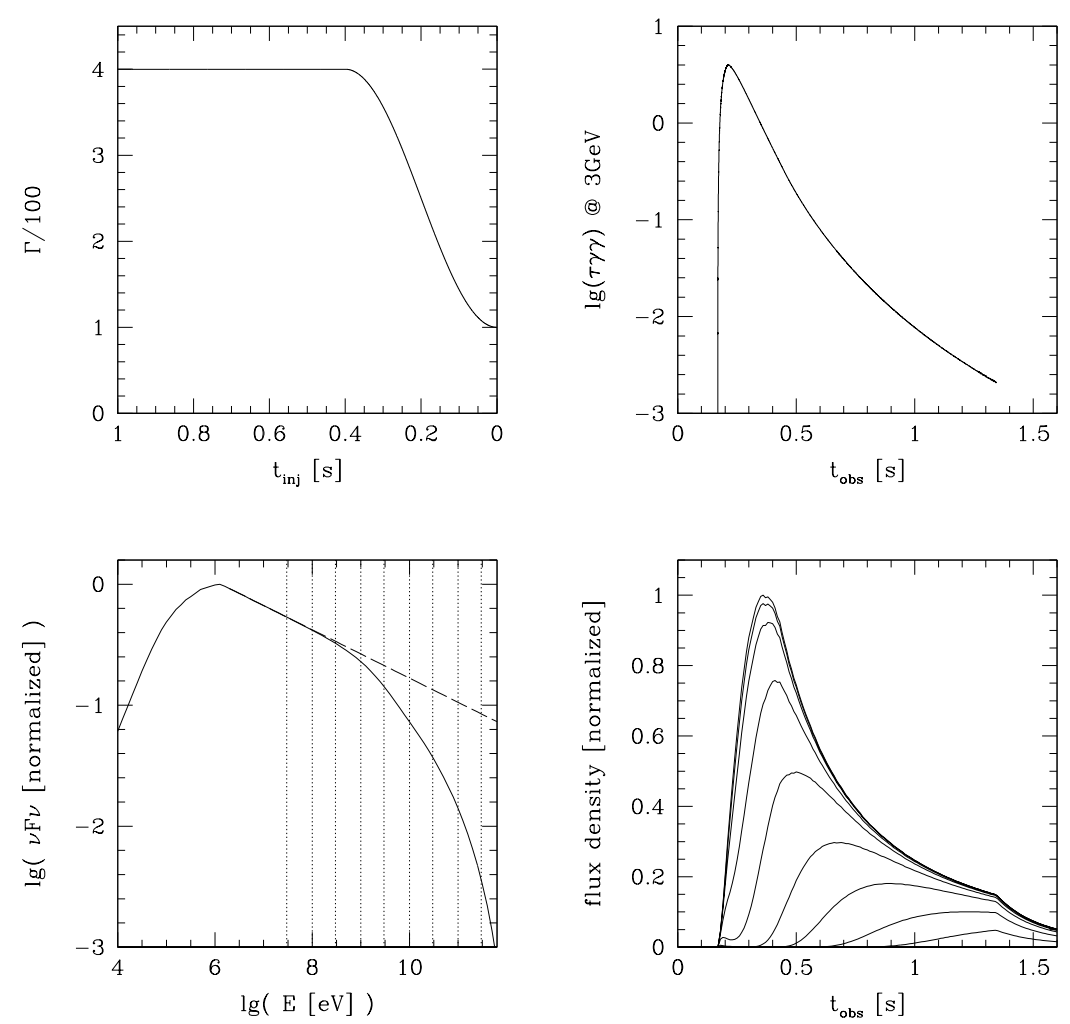

Figure 2: Delayed onset produced by the $\gamma \gamma$ opacity. This set of figures illustrates with a synthetic burst how the temporal evolution of the $\gamma \gamma$ opacity can induce naturally a delay onset for the high energy emission within the internal shock framework. Upper left panel: initial Lorentz factor distribution of the outflow as a function of the injection time $t_{i n j}$. Upper right panel: the evolution of the $\gamma \gamma$ opacity seen by $3 \mathrm{GeV}$ photons emitted on the line of sight as a function of the time $t_{o b s}$ at which they are received by the observer. Lower right panel: light curves calculated at different photon energies $(30 \mathrm{MeV} ; 100 \mathrm{MeV} ; 300 \mathrm{MeV} ; 1 \mathrm{GeV} ; 3$ $\mathrm{GeV} ; 10 \mathrm{GeV} ; 30 \mathrm{GeV} ; 100 \mathrm{GeV} ; 300 \mathrm{GeV}$ ). Lower left panel: spectrum integrated over the whole duration of the burst. The vertical dotted lines mark the photon energies at which light curves are calculated.

where the successive collisions between shells mimic the propagation of shock waves [7]. Each collision produces an elementary spherical flash: the simulated light curves are the result of the sum of all flashes. For each high energy photon, the $\gamma \gamma$ opacity is computed by integrating equation (1) from its emission location to the observer taking into account the exact radiation field $n_{\Omega}$ produced by all the collisions in the outflow. A previous study of the $\gamma \gamma$ opacity in internal shock was made by [8]. However the prescription used to compute $\tau_{\gamma \gamma}$ was still approximate, using the local physical conditions of the outflow where the high energy photon is emitted and applying them to an average formula of $\tau_{\gamma \gamma}$ (as can be found in $[2,4,5]$ ).

\subsection{Is the delayed onset of the $\mathrm{GeV}$ emission a signature of the $\gamma \gamma$ opacity ?}

The high energy emission (above $100 \mathrm{MeV}$ ) detected by Fermi in a few GRBs has often a delayed onset compared to the softer $\gamma$-ray emission (below $5 \mathrm{MeV}$ ). This feature seems to be 
common to long and short GRB classes and its origin is still debated [11]. Among the proposed explanations the possibility that it is induced by a $\gamma \gamma$ opacity temporal evolution effect was already discussed [4]. The main idea of this proposition is to consider that as the shock wave producing the $\gamma$-ray emission expands to larger radii the opacity seen by the emitted high energy photons evolve from an optically thick to an optically thin regime. The model developed in the present study is appropriate to investigate this possibility in more details. Figure 2 illustrates an example of a synthetic burst displaying a delay between the high energy and the soft $\gamma$-ray emissions. The initial Lorentz factor distribution of the relativistic outflow is simple with the formation of two shocks when the fast part of the ejecta catches up with the slow part: an internal "forward" shock sweeping the slow part and an internal "reverse" shock sweeping the fast part. The internal "forward" shock quickly disappears and contributes to the observed $\gamma$-ray emission only at early times $\left(t_{o b s}<0.21\right.$ $\mathrm{s})$. On the other hand the internal "reverse" shock has a longer duration and expands from a radius $R_{b}=8 \cdot 10^{13} \mathrm{~cm}$ to a radius $R_{e}=3 \cdot 10^{15} \mathrm{~cm}$ during its $\gamma$-ray emission activity. Due to the increase of the emission radius of the shock the $\gamma \gamma$ opacity at a given frequency is smaller for photons received at later times. This example shows that the temporal evolution of the $\gamma \gamma$ opacity can actually induce a significant delay between the high energy and the soft $\gamma$-ray emissions.

Testing this theoretical idea for the observed delay remains difficult as the characteristics of the high-energy $\gamma$-ray spectrum in Fermi-LAT GRBs remain unclear. The observed time integrated spectra often keep consistent with a unique Band function component over the GBM and LAT energy ranges (e.g. GRB 080916C), whereas the lightcurves suggest a different behavior at high energy. It is very likely that there is an additional component at high energy which is however too weak in most cases to be clearly identified in the spectral analysis (an additional high energy component was detected in the case of GRB 090510 [5]). Then, the observed evolution of the high energy slope $\beta$ in the Band function could be real, or related to the evolution of this weak component. Clearly, despite a very significant improvement compared to previous instruments, Fermi-LAT cannot yet provide a detailed picture of the high energy part of the spectrum where several effects are expected to superimpose: shape of the high-energy tail of the main component, location and shape of the additional weak high energy component, location and shape of the $\gamma \gamma$ attenuation.

An interesting prediction of such a scenario is that the onset delay should increase with frequency since the transparency regime is reached at larger radii (and so at larger observer times) for photons of higher energies. However the paucity of photons at high energy does not allow to strongly constrain this potential behavior. Finally it was argued $[6,4]$ that in such a situation a high energy emission should also be detected at the very beginning of the burst while the photon field inducing the $\gamma \gamma$ opacity is still building up. However this early transparency phase is very short (of the order of $\Delta t_{o b s} \approx 100 \mathrm{~ms}$ for the synthetic burst presented here) and occurs when the $\gamma$-ray emission is still at the beginning of its onset rise. It is not clear if the current data can put significant constraints on such a possible feature.

\subsection{Minimum Lorentz factor: detailed modeling vs. simple estimates.}

One natural application of our model is the estimate of the minimum bulk Lorentz factor $\Gamma_{\min }$ in GRB outflows. Our model reproduces the dependence of $\tau_{\gamma \gamma}$ on the mean Lorentz factor $\bar{\Gamma}$ of the outflow and the energy of the high energy photon $E_{G e V}: \tau_{\gamma \gamma} \propto \Gamma^{2(\beta-1)} E_{G e V}^{-(1+\beta)}$. However the 

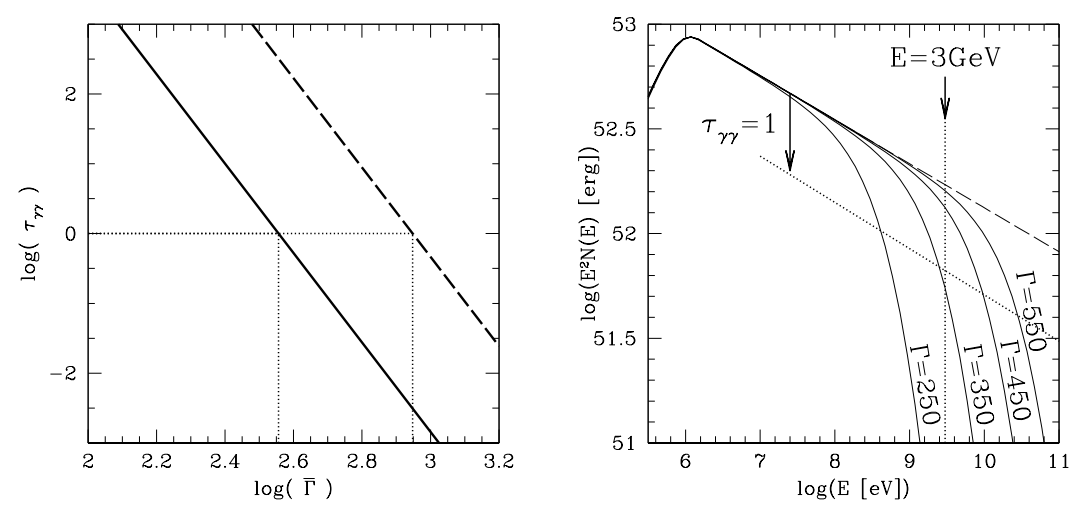

Figure 3: Minimum Lorentz factor for GRB 080916C. Left panel: evolution of $\tau_{\gamma \gamma}$ against the mean Lorentz factor of the outflow $\bar{\Gamma}$ following our detailed modeling (solid line) and using an average formula [4] (dashed line). Right panel: time-integrated spectra obtained for different mean Lorentz factor of the outflow (the shape of the initial Lorentz factor distribution stays the same) and the reference spectrum without $\gamma \gamma$ annihilation (dashed line).

normalization we obtain is different compared to a simple "single zone" model (see fig. 3, left panel) and gives values of $\Gamma_{\min }$ which are lower by a factor of 2-3 (the exact factor depending on the details of the dynamical features of the GRB). To illustrate this aspect with an example our approach was applied to the case of GRB 080916C. Using our numerical model a synthetic GRB was generated which reproduces the main observational characteristics of the GRB: the total radiated isotropic $\gamma$-ray energy $\left(E_{i s o}=8.8 \times 10^{54} \mathrm{ergs}\right)$, the spectral properties $\left(E_{p}, \alpha, \beta\right.$ parameters of the Band function), the envelop of the light curve and a short time-scale variability of $2 \mathrm{~s}$ in the observer frame. The study is focused on the most constraining time bin (time bin $b$ ), during which the highest observed photon energy was $3 \mathrm{GeV}\left(16 \mathrm{GeV}\right.$ in the source rest frame). $\Gamma_{\min }$ is obtained by requiring that for this photon $\tau_{\gamma \gamma}<1$ (see figure 3, right panel). Following our approach we find a minimum Lorentz factor $\Gamma_{\min }=360$, instead of 887 which was obtained from an approximate "single zone" model [4].

\section{Conclusions}

The detailed $\gamma \gamma$ opacity calculation model presented in these proceedings is appropriate and accurate to study many aspects and consequences of $\gamma \gamma$ annihilation in GRBs. In the present work we focus on the internal shock model and consider the consequences and signatures that $\gamma \gamma$ opacity could have in GRB observations. The model was validated by comparing our results to a previous semi-analytical study [6].

- It is shown how a detailed calculation can predict minimum Lorentz factors $\Gamma_{\min }$ which are lower by a factor of 2-3 compared to a simplified "single zone" model where spatial and temporal dependencies are averaged out.

- The temporal evolution of $\tau_{\gamma \gamma}$ during a burst could favor a delay between the $\mathrm{MeV}$ and GeV light curves. 
Other effects (Hascoët et al. in prep.) can be studied with our model:

- The $\gamma \gamma$ cutoff transition can be characterized in time-integrated spectra. It is usually closer to a power-law steepening than to a sharp exponential cutoff. The exact shape of the transition strongly depends on the details of the GRB dynamics.

- For complex GRBs, the $\gamma \gamma$ opacity could suppress the shortest time-scale features in high energy light curves (above $100 \mathrm{MeV}$ ).

- If $\mathrm{MeV}$ and $\mathrm{GeV}$ photons are not produced at the same location (see e.g. $[9,10]), \tau_{\gamma \gamma}$ could be further lowered, reducing even more the constraint on $\Gamma_{\min }$.

\section{References}

[1] M. J. Rees, Appearance of Relativistically Expanding Radio Sources, Nature 211, 468-470 (1966).

[2] Y. Lithwick, and R. Sari, Lower Limits on Lorentz Factors in Gamma-Ray Bursts, ApJ 555, 540-545 (2001), [astro-ph/0011508].

[3] J. Bregeon, A. Goldstein, R. Preece, et al., Detection of a spectral break in the extra hard component of GRB 090926A, ArXiv e-prints (2011), ast ro-ph/1101.2082.

[4] A. A. Abdo, M. Ackermann, M. Arimoto, et al., Fermi Observations of High-Energy Gamma-Ray Emission from GRB 080916C, Science 323, 1688- (2009).

[5] M. Ackermann, K. Asano, W. B. Atwood, et al., Fermi Observations of GRB 090510: A Short-Hard Gamma-ray Burst with an Additional, Hard Power-law Component from $10 \mathrm{keV}$ TO GeV Energies, ApJ 716, 1178-1190 (2010).

[6] J. Granot, J. Cohen-Tanugi, and E. do Couto e Silva, Opacity Buildup in Impulsive Relativistic Sources, ApJ 677, 92-126 (2008), [astro-ph/ 0708 . 4228].

[7] F. Daigne, and R. Mochkovitch, Gamma-ray bursts from internal shocks in a relativistic wind: temporal and spectral properties, MNRAS 296, 275-286 (1998), [astro-ph/9801245].

[8] J. Aoi, K. Murase, K. Takahashi, et al., Can We Probe the Lorentz Factor of Gamma-ray Bursts from GeV-TeV Spectra Integrated Over Internal Shocks?, ApJ 722, 440-451 (2010), [astro-ph/0904.4878].

[9] Ž. Bošnjak, F. Daigne, and G. Dubus, Prompt high-energy emission from gamma-ray bursts in the internal shock model, A\&A 498, 677-703 (2009), [astro-ph / 0811 .2956].

[10] Y. Zou, Y. Fan, and T. Piran, A Revised Limit of the Lorentz Factors of Gamma-ray Bursts with Two Emitting Regions, ApJL 726, L2+ (2011), [astro-ph/1008.2253].

[11] J. Granot, for the Fermi LAT Collaboration, the GBM Collaboration, Highlights from Fermi GRB observations, in the Proceedings of The Shocking Universe - Gamma-Ray Bursts and High Energy Shock phenomena (2010), [astro-ph/1003.2452].

[12] R. Hascoët, V. Vennin, F. Daigne, R. Mochkovitch, Do Fermi-LAT observations really imply very large Lorentz factors in GRB outflows ?, in the Proceedings of the Gamma Ray Bursts 2010 Conference (2011), [astro-ph/1101.3889]. 